\title{
Dissolution of Expanded Polystyrene in Cycloalkane Solutions
}

\author{
Shiying Tao1,2, Boyang $\mathrm{Li}^{3}$, Hui Huang ${ }^{3 *}$ \\ ${ }^{1}$ Faculty of Science and Engineering, The University of Nottingham Ningbo, Ningbo, China \\ ${ }^{2}$ Ningbo Nottingham New Materials Institute Ltd., Ningbo, China \\ ${ }^{3}$ School of Materials and Chemical Engineering, Ningbo University of Technology, Ningbo, China \\ Email: ^huihuang@nbut.edu.cn
}

How to cite this paper: Tao, S.Y., Li, B.Y. and Huang, H. (2018) Dissolution of Expanded Polystyrene in Cycloalkane Solutions. Open Journal of Applied Sciences, 8, 465-471.

https://doi.org/10.4236/ojapps.2018.810037

Received: September 30, 2018

Accepted: October 23, 2018

Published: October 26, 2018

Copyright $\odot 2018$ by authors and Scientific Research Publishing Inc. This work is licensed under the Creative Commons Attribution International License (CC BY 4.0). http://creativecommons.org/licenses/by/4.0/

\begin{abstract}
Feasibility of dissolution and utilization of expanded polystyrene in cycloalkane solutions was investigated in this work. The dissolution process of expanded polystyrene in several cycloalkane solutions decalin, cyclohexane and methyl cyclohexane was studied. The effect of dissolution temperature, mechanical agitation, ultrasonic wave and stirring rate was studied under optimized conditions. Mass transfer coefficients were fitted. The results showed that the dissolution rate of expanded polystyrene in different cycloalkane solutions was ranked as decalin > methyl cyclohexane > cyclohexane; higher dissolution temperature and faster stirring rate could speed up the dissolution of expanded polystyrene; the effect of mechanical agitation was superior to ultrasonic condition; the solubility of top face was better than side face and under face.
\end{abstract}

\section{Keywords}

Expanded Polystyrene, Cycloalkane, Dissolution, Mass Transfer Coefficient

\section{Introduction}

Polystyrene is one of the general plastics. It is widely used in packaging container materials, insulation materials, daily necessities, electrical and electronic fields because of its high transparency, good insulation performance, water resistance, corrosion resistance, easy processing and molding. However, the discarded polystyrene products cause white pollution to the environment. China is facing the problems of large amount of waste foamed polystyrene (EPS) and low recycling efficiency of it [1]. Therefore, the recycling of EPS has always been one of the hot issues concerned by scholars, and solvent regeneration is one of the important 
recycling ways [2]-[7]. A new material, polycyclohexyl ethylene, can be obtained by catalytic hydrogenation after polystyrene is dissolved in solvents [8] [9] [10]. In this paper, the dissolution process of EPS was studied indifferent cycloalkane solvents, like decalin, cyclohexane and methylcyclohexane which were frequently used as solvent system in the catalytic hydrogenation of polystyrene. It is desirable to provide theoretical support for the development of high value-added polycyclohexane ethylene (PCE) by direct catalytic hydrogenation from waste EPS after dissolution.

\section{Experimental}

\subsection{Materials and Methods}

EPS was obtained from the local market. Decalin, cyclohexane and methylcyclohexane with reagent grade were bought from Sinopharm Chemical Reagent Co., Ltd (Shanghai, China). The concentration of polystyrene was determined with a UV-7504C UV-vis Spectro-photometer at $261.5 \mathrm{~nm}$. The ultrasonic wave was generated by BL10-250A ultrasonic generator.

\subsection{Standard Curve of Polystyrene}

The EPS was dissolved in different cycloalkane solutions and diluted to form solutions with different polystyrene concentrations. The data of the concentration (c) of polystyrene and absorbance (a) were fitted to a standard curve, namely $a=2.39702 c-0.02258(\mathrm{R}=0.99967)$.

\subsection{Dissolution Process}

A certain amount of cycloalkane solution was put into a beaker and then the beaker was placed in a water bath with stirring and temperature control device. EPS was made into a rectangular strip with a cross-sectional area of $2 \mathrm{~cm} \times 2 \mathrm{~cm}$ and inserted vertically into the solvent (cycloalkane solution). After isolation, only one side of the EPS was in contact with the solvent in the dissolution process. Sampling and analysis were carried out to calculate the thickness (d) of the EPS.

\subsection{Dissolution Coefficient}

The thickness of a single EPS face would decrease in the process of dissolution. In this condition, mass transfer is the rate control step. The dissolution rate per unit cross-sectional area of EPS is expressed as:

$$
-\frac{1}{A} \frac{\mathrm{d} W}{\mathrm{~d} t}=-\rho_{s} \frac{\mathrm{d} L}{\mathrm{~d} t}=k \Delta C
$$

where $A$ is the cross-sectional area of the solvent surface in the unit of $\mathrm{cm}^{2}, W$ is the weight of EPS in the unit of $\mathrm{g}, L$ is the thickness of EPS in the unit of $\mathrm{cm}, \rho_{\mathrm{s}}$ is the density of EPS in the unit of $\mathrm{g} / \mathrm{cm}^{3}, \Delta C$ is the difference between the saturation concentration of solute in the sphere and the overall concentration in the solvent in the unit of $\mathrm{mg} / \mathrm{ml}, k$ is a constant. 
In the initial condition of $t=0$ and $d=0$, the expression of (1) could be rewritten as:

$$
d=\frac{k \Delta C}{\rho_{s}} t
$$

where $d$ has a linear relationship with $t$, and the slope is mass transfer coefficient (m).

\section{Results and Discussion}

\subsection{Effect of Solvent on EPS Dissolution}

EPS strips were dissolved in three cycloalkane solvents (decalin, cyclohexane and methylcyclohexane) at the temperature of $25^{\circ} \mathrm{C}$ and stirring rate of $200 \mathrm{rpm}$, respectively. The effect of solvent on EPS dissolution strips is shown in Figure 1.

As shown in Figure 1, the dissolution rate of EPS in the three solvents increases linearly in the first 4 minutes, and then gradually become constant, respectively. It can be seen that the dissolution rates of EPS in the three solvents are in the order of decalin $(m=0.19525)>$ methyl cyclohexane $(m=0.13650)>$ cyclohexane $(m=0.11372)$. A large number of bubbles rise during the dissolution process, which will adhere to the dissolving face of EPS, preventing the dissolution process. Meanwhile, the increment of the distance between the blades of the mixer and the dissolving face weaks the influence of the stirring on the dissolution. So the dissolution curve gradually becomes stable. Decalin was used as solvent for subsequent experiments as the dissolution rate of EPS in it is higher than that of the other two solvents.

\subsection{Effect of Temperature on EPS Dissolution}

The dissolution of EPS at temperatures of $15^{\circ} \mathrm{C}, 25^{\circ} \mathrm{C}$ and $35^{\circ} \mathrm{C}$ was carried outin $300 \mathrm{ml}$ of decal insolvent with stirring rate of $200 \mathrm{rpm}$. The experimental result is shown in Figure 2.

The temperature has a significant impact on dissolution rate. It is observed that there is an obvious increase of dissolved thickness of EPS at the temperature

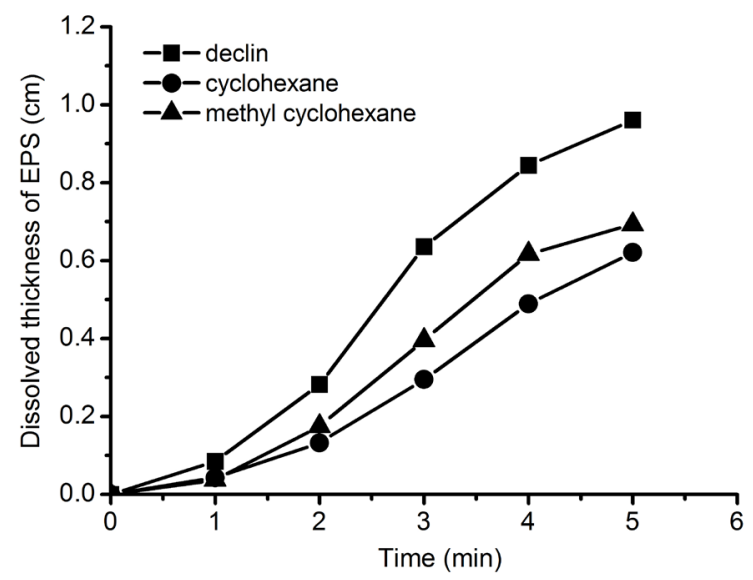

Figure 1. Effect of different cycloalkane solutions on EPS dissolution. 


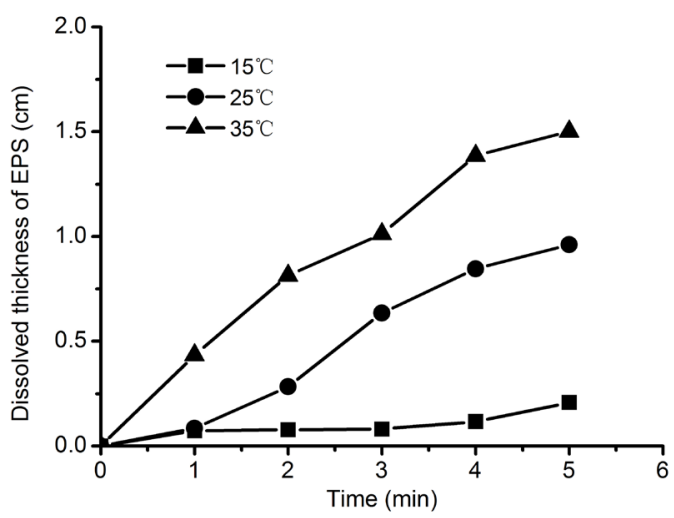

Figure 2. Effect of dissolution temperature on EPS dissolution.

of $25^{\circ} \mathrm{C}$ and $35^{\circ} \mathrm{C}$. The smooth curve of $15^{\circ} \mathrm{C}$ indicats that low temperature is not good for EPS dissolving. The effect of temperature was confirmed by the value of tree mass transfer coefficients, $m=0.03582,0.19525$ and 0.32996 , representing for that of $15^{\circ} \mathrm{C}, 25^{\circ} \mathrm{C}$ and $35^{\circ} \mathrm{C}$, respectively.

\subsection{Effect of Ultrasonic Wave on EPS Dissolution}

To compare the effect of mechanical agitation andultrasonic wave on EPS dissolution. The dissolution process was conducted in $300 \mathrm{ml}$ of decal insolvent at temperature of $25^{\circ} \mathrm{C}$. The result is shown in Figure 3.

At the same condition, the dissolution curve of mechanical stirring indicated a faster dissolution rate $(m=0.19525)$ than that of ultrasonic wave $(m=0.10404)$. This could be attributed to the fact that the bubble appeared in the ultrasonic wave-assisted dissolution process attached finely to the dissolution interface, which impeded the mass transfer.

\subsection{Effect of Stirring Rate on EPS Dissolution}

The EPS dissolution at stirring rate of $200 \mathrm{rpm}, 300 \mathrm{rpm}$ and $400 \mathrm{rpm}$ was studied in $300 \mathrm{ml}$ decalin solvent at $25^{\circ} \mathrm{C}$. The effect of stirring rate on EPS dissolution is shown in Figure 4.

The dissolution rate of EPS increased steadily with dissolution time, and higher dissolution rate appeared at the faster stirring rate. The fitted mass transfer coefficients for three dissolution process is 0.19525 (200 rpm), 0.22792 $(300 \mathrm{rpm})$ and $0.38521(400 \mathrm{rpm})$, respectively. The transition of the curve of $200 \mathrm{rpm}$ after 3 minutes indicates that low stirring rate could not cause enough disturbance to the EPS dissolution. It is more difficult to dispel the attached bubbles attached at the lower stirring rate as the dissolving face gradually become farther from the stirring blade.

\subsection{Effect of Dissolution Face on EPS Dissolution}

The effect of dissolution face on EPS dissolution was conducted under temperature of $25^{\circ} \mathrm{C}$ and stirring rate of $200 \mathrm{rpm}$ in $300 \mathrm{ml}$ of decal insolvent. The result is shown in Figure 5. It is found that the dissolution rate increases similarly for 


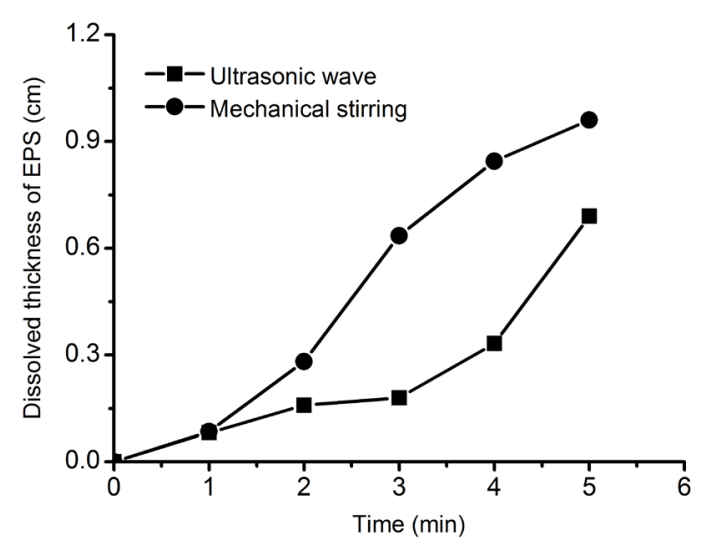

Figure 3. Effect of ultrasonic wave on EPS dissolution.

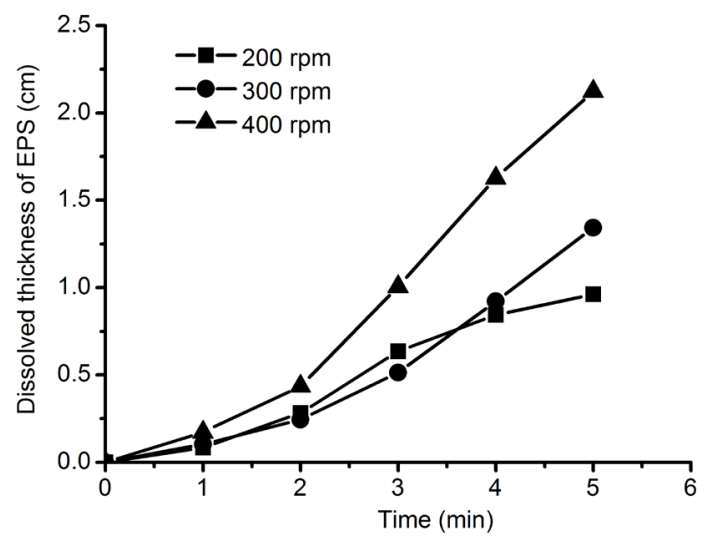

Figure 4. Effect of stirring rate on EPS dissolution.

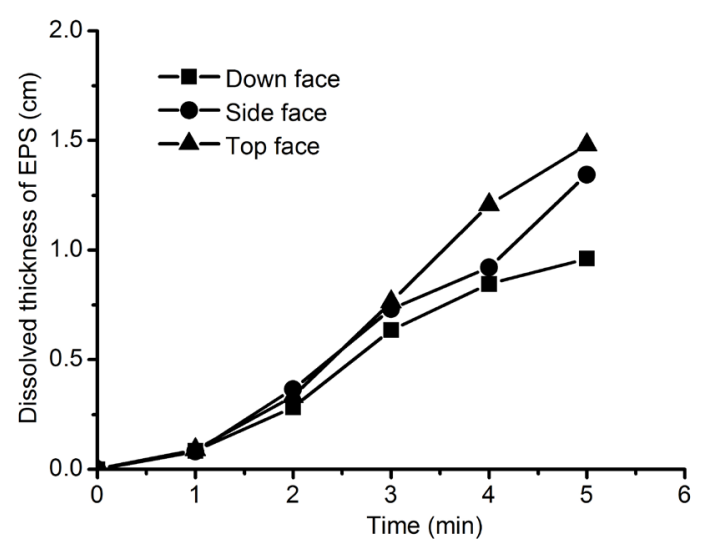

Figure 5. Effect of dissolution face on EPS dissolution.

the three faces in the first 3 minutes. However, the dissolution rate increases higher for the upper face in the stage of 3 to 5 minute. This trend can be explained by the observation that the number of attached bubbles to the upper face is less than that of the down face. While disturbance generated by bubbles in the process of upward floating could enhance mass transfer of the side face and top face of EPS. The mass transfer coefficient $(m)$ of three dissolving face are 0.19525 (down face), 0.24372 (side face) and 0.27775 (top face), respectively. 


\section{Conclusion}

The dissolution process of expanded polystyrene in three cycloalkane solutions (decalin, cyclohexane and methyl cyclohexane) was studied in this work. The results reveals that the dissolution rate of expanded polystyrene in decalin is the fastest with the solubility mass transfer coefficient of 0.19525 under temperature of $25^{\circ} \mathrm{C}$ and stirring rate of $200 \mathrm{rpm}$, when compared with that of methyl cyclohexane, 0.13650 , and cyclohexane, 0.11372 . The solubility mass transfer coefficient increases with temperature in the range of $15^{\circ} \mathrm{C}$ to $35^{\circ} \mathrm{C}$, and reached the maximum of 0.32996 . The solubility mass transfer coefficient increases with stirring rate in the range of $200 \mathrm{rpm}$ to $400 \mathrm{rpm}$, and reached the maximum of 0.38521 . Influenced by bubbles generated in the dissolution process, the dissolution rate under ultrasonic wave condition showed no superiority to that of mechanical stirring condition. At the temperature of $25^{\circ} \mathrm{C}$ and stirring rate of $200 \mathrm{rpm}$, the solubility mass transfer coefficient was $0.27775,0.24372$ and 0.19525 , with the dissolution surface of upper face, side face and under face, respectively.

\section{Acknowledgements}

This work is financially supported by Zhejiang Xinmiao Talent Program (2018R428012) and Ningbo Natural Science Foundation (2017A610066).

\section{Conflicts of Interest}

The authors declare no conflicts of interest regarding the publication of this paper.

\section{References}

[1] Zhang, W.M., Zhou, J.T., Li, T., Yang, B.L. and Huang, H. (2015) Reutilization of Expanded Polystyrene Waste. Journal of Ningbo University of Technology, 27, 11-14.

[2] Jiménez, E., Cabañas, B. and Lefebvre, G. (2014) Environment, Energy and Climate Change I. The Handbook of Environmental Chemistry. Springer, Cham.

[3] Yao, M., Ning, T.M., Peng, T.K. and Jiang, Z.G. (2017) Recycling of Waste Expanded Polystyrene (EPS) by Graft Polymerization. Journal of Beijing University of Chemical Technology, 44, 28-33.

[4] Gutiérrez, C., García, M.T., Gracia, I., Lucas, A.D. and Rodríguez, J.F. (2013) The Selective Dissolution Technique as Initial Step for Polystyrene Recycling. Waste and Biomass Valorization, 4, 29-36. https://doi.org/10.1007/s12649-012-9131-9

[5] Liu, D. and Liu, J.L. (2014) Converting Waste Expanded Polystyrene into Polymeric Azo Dyes Containing the Sulfonamide Group. Journal of Material Cycles and Waste Management, 16, 557-565. https://doi.org/10.1007/s10163-013-0210-1

[6] Huang, J., Yin, X.Y., Yang, J.Y. and Guo, M.L. (2014) Solid Protonic Acids and Luminescent Carbon Dots Derived from Waste Expanded Polystyrene. Materials Letters, 117, 112-115. https://doi.org/10.1016/j.matlet.2013.11.104

[7] Yang, S.J. and Yang, Q. (2012) Study on Process of Recovering Polystyrene with Solvent Method. Modern Chemical Industry, 32, 43-45.

[8] Huang, H., Zhou, J.T., Ying, Q.R. and Tao, S.Y. (2014) Preparation of Magnetic 
Palladium Catalyst and Its Kinetic Study of Polystyrene Hydrogenation. Modern Chemical Industry, 34, 70-73.

[9] Huang, H., Fan, Y.F., Tao, S.Y. and Gao, H.Q. (2013) Progress in Catalytic Hydrogenation of Polystyrene to Poly(Cyclohexylethylene). New Chemical Materials, 41, 178-179.

[10] Li, C.Y., Feng, M., Cui, H.F., Cao, G.P., Lv, H. and Chen R.Q. (2017) Preparation of Carbon Nanotube Catalyst on Structure-Modified Cordierite Monolith for Polystyrene Hydrogenation. Journal of Chemical Industry and Engineering (China), 68, 2746-2754. 